\title{
The use of filler DNA for improved transfection and reduced DNA needs in transient gene expression with $\mathrm{CHO}$ and HEK cells
}

\author{
Divor Kiseljak, Yashas Rajendra, Sagar S Manoli, Lucia Baldi, David L Hacker, Florian M Wurm* \\ From 22nd European Society for Animal Cell Technology (ESACT) Meeting on Cell Based Technologies \\ Vienna, Austria. 15-18 May 2011
}

\section{Background}

Transient gene expression (TGE) is a rapid method for the production of recombinant proteins. Protein productivity in TGE has improved significantly over the past decade, reaching $300 \mathrm{mg} / \mathrm{L}$ and $1 \mathrm{~g} / \mathrm{L}$ in CHO DG44 (CHO) and HEK 293E (HEK) cells, respectively [1,2]. However, the amount of plasmid DNA needed for transfection remains relatively high, contributing significantly to the overall cost of the TGE process. In order to reduce the amount of plasmid DNA in TGE, we examined the possibility of partially replacing it with herring sperm DNA (non-coding "filler" DNA) in transfections of $\mathrm{CHO}$ and HEK cells.

\section{Materials and methods Transfections}

Suspension-adapted $\mathrm{CHO}$ were centrifuged and resuspended in ProCHO5 (Lonza, Verviers, Belgium) at a density of $4 \times 10^{6}$ cells $/ \mathrm{mL}$. Transfections of $5 \mathrm{~mL}$ were performed in TubeSpin ${ }^{\circledR} 50$ bioreactors (TPP, Trasadingen, Switzerland) using $0.625 \mu \mathrm{g}$ DNA $/ 1 \times 10^{6}$ cells and $2.5 \mu \mathrm{g} / 1 \times 10^{6}$ cells of linear $25 \mathrm{kDa}$ polyethyleneimine (PEI; Polysciences, Eppenheim, Germany). pA3 carrying the genes for a human IgG light and heavy chains was used for transfections [1]. The transfected cultures were incubated at $31{ }^{\circ} \mathrm{C}$ in $5 \% \mathrm{CO}_{2}$ and $85 \%$ humidity with agitation at $180 \mathrm{rpm}$. HEK cells were centrifuged and resuspended at a density of $20 \times 10^{6}$ cells $/ \mathrm{mL}$ in RPMI 1640 medium (Lonza). To each culture, $1.0 \mu \mathrm{g}$ DNA/ $1 \times 10^{6}$ cells and $3.0 \mu \mathrm{g}$ PEI $/ 1 \times 10^{6}$ cells were added. At 3 $\mathrm{h}$ post-transfection, cells were diluted with ExCell293 $^{\mathrm{TM}}$ medium (Sigma) medium to a density of $1 \mathrm{x}$

\footnotetext{
* Correspondence: florian.wurm@epfl.ch

Laboratory for Cellular Biotechnology, Faculty of Life Sciences, École

Polytechnique Fédéral de Lausanne, CH-1015 Lausanne, Switzerland
}

$10^{6}$ cells $/ \mathrm{mL}$, and valproic acid was added to a final concentration of $3.75 \mathrm{mM}$. The transfected cultures were incubated at $37^{\circ} \mathrm{C}$ as above.

\section{Analyses}

The IgG concentration was determined by sandwich ELISA [3]. To quantify plasmid DNA copy number, total cellular DNA was extracted using DNeasy Blood \& Tissue Kit (Qiagen) according to the manufacturer's protocol. To estimate the mRNA transgene levels, total RNA was extracted from cells using the GenElute mRNA kit (Sigma) according to the manufacturer's protocol. DNA-free RNA was reverse transcribed using MMLV reverse transcriptase (Sigma) and oligo dT as the primer. The RT-qPCR was carried out in a LightCycler 480 Real-Time PCR System (Roche Applied Science, Basel, Switzerland) with the ABsolute QPCR SYBR Green ROX mix (Thermo Fisher Scientific) according to the manufacturer's instructions.

\section{Results}

Filler DNA allows considerable reduction in coding pDNA amounts

We tested the efficiency of herring sperm DNA as filler for TGE in CHO and HEK cells. We reduced the amount of pA3 to $17 \%$ or $33 \%$ of the optimum amount for each cell line and added filler DNA to $100 \%$. The total amount of PEI was kept constant for all conditions. We observed that antibody titers increased when filler DNA was co-transfected with pA3 as compared to transfection with a reduced amount of pA3 alone (Fig. 1). These results showed that up to $83 \%$ of the coding pDNA could be replaced by filler DNA with only a minimal negative impact on yield. 

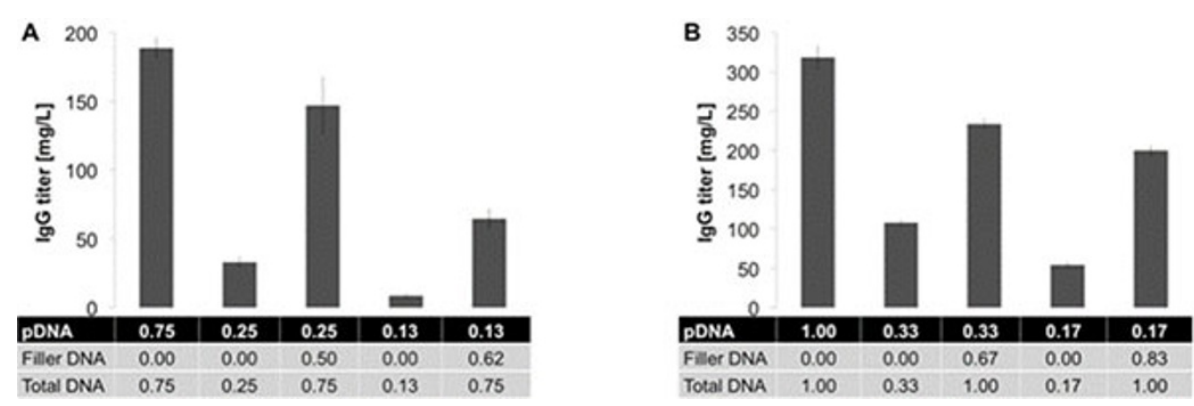

Figure 1 Effect of filler DNA on transient lgG production in A) CHO and B) HEK cells. IgG titers were measured on day 7 post-transfection by ELISA. The DNA amounts are presented in $\mu \mathrm{g} / 10^{6}$ cells.

\section{Filler DNA does not influence the delivery of coding pDNA}

We investigated whether the use of filler DNA could improve pDNA delivery and/or intracellular pDNA stability by quantifying the plasmid copy number by qRTPCR. The results showed that the plasmid copy number decreased proportionally with the amount of pA3 transfected in the presence or absence of filler DNA in both CHO and HEK cells (data not shown). Therefore, filler DNA did not influence the delivery of coding pDNA to transfected cells.

\section{Filler DNA enhances transgene mRNA levels and improves release of coding pDNA}

We tested the hypothesis that the use of filler DNA could influence transcriptional competence of coding pDNA. By qPCR, we found that transgene mRNA levels were significantly higher in the presence of filler than in its absence (data not shown). This may explain the improved protein titers observed in the presence of filler DNA. We then hypothesized that filler DNA could influence the strength of PEI:DNA complexes and pDNA release from the complex upon delivery. With an in vitro dextran sulfate displacement assay we observed that with increasing PEI:DNA ratios, complex strength increased. However, when filler DNA was added to the complex, the release of pDNA from the complex was improved (data not shown).

\section{Conclusions}

Our data show that in TGE the amount of the coding vector could be reduced considerably by replacement of a significant proportion of pDNA with filler DNA (herring sperm DNA) without a major negative impact on recombinant protein productivity. However, filler DNA did not influence the delivery or stability of pDNA. The addition of filler DNA to the DNA-PEI complex, however, relaxed the complex in vitro. Based on these results, we speculate that the presence of filler DNA results in a more efficient intracellular release of the
pDNA from the DNA-PEI complex and thus to improved transgene transcription.

\section{Acknowledgments}

This work has been supported in part by the CTI Innovation Promotion Agency of the Swiss Federal Department of Economic Affairs ( $n$. 10563.1PFLS-LS) under a collaboration with ExcellGene SA (Monthey, Switzerland). TPP (Trasadingen, Switzerland) is acknowledged for providing TubeSpin ${ }^{\circledR}$ bioreactor 50 tubes.

Published: 22 November 2011

\section{References}

1. Rajendra Y, Kiseljak D, Baldi L, Hacker D, Wurm FM: A simple high-yielding process for transient gene expression in CHO cells. J Biotechnol 2011, 153:22-26

2. Backliwal $G$, Hildinger $M$, Chenuet $S$, Wulhfard $S$, De Jesus M, Wurm FM: Rational vector design and multi-pathway modulation of HEK $293 \mathrm{E}$ cells yield recombinant antibody titers exceeding $1 \mathrm{~g} / \mathrm{l}$ by transient transfection under serum-free conditions. Nuc Acid Res 2008, 36(15)

3. Meissner P, Pick H, Kulangara A, Chatellard P, Friedrich K, Wurm FM: Transient gene expression: recombinant protein production with suspension-adapted HEK293-EBNA cells. Biotechnol Bioeng 2001, 75:197-203.

doi:10.1186/1753-6561-5-S8-P33

Cite this article as: Kiseljak et al:: The use of filler DNA for improved transfection and reduced DNA needs in transient gene expression with CHO and HEK cells. BMC Proceedings 2011 5(Suppl 8):P33.

\section{Submit your next manuscript to BioMed Central} and take full advantage of:

- Convenient online submission

- Thorough peer review

- No space constraints or color figure charges

- Immediate publication on acceptance

- Inclusion in PubMed, CAS, Scopus and Google Scholar

- Research which is freely available for redistribution 